\title{
An update on immunotherapy in breast cancer
}

\author{
Rupert Bartsch $(\mathbb{D} \cdot$ Elisabeth Bergen · Arik Galid
}

Received: 7 January 2019 / Accepted: 15 January 2019 / Published online: 23 January 2019

(C) The Author(s) 2019

Summary While immunotherapy (IOT) with monoclonal antibodies has long been present in HER2positive breast cancer, the development of modern IOT concepts such as PD-1/PD-L1 targeting immune checkpoint inhibitors has been slow compared with other malignancies such a melanoma or lung cancer. Recent clinical trials of IOT have focused on triple-negative breast cancer (TNBC) as no specific treatment options beyond chemotherapy have been available in this subtype; in addition, TNBC apparently harbours the largest immunogenic potential. Meanwhile, initial results from the phase III IMpassion130 trial have been presented; here, the addition of atezolizumab to nab-paclitaxel led to a clinically meaningful prolongation of overall survival in the PD-L1 positive subset, potentially defining a novel standard-of-care in the first-line treatment of TNBC. Further evaluation of checkpoint inhibitors alone or in combination with chemotherapy or targeted drugs are currently ongoing in TNBC as well as in other breast cancer subtypes and clinical development is also ongoing in the adjuvant and neoadjuvant settings. This short review summarizes results of recent trials with a focus on clinical outcome data and discusses the ongoing development of IOT in breast cancer.

Keywords Atezolizumab - Breast cancer - Immune checkpoint inhibitors $\cdot$ IMpassion $130 \cdot$ Pembrolizumab

\section{R. Bartsch $(\bowtie) \cdot$ E. Bergen}

Department of Medicine 1, Clinical Division

of Oncology, Medical University of Vienna,

Waehringer Guertel 18-20, 1090 Vienna, Austria

rupert.bartsch@meduniwien.ac.at

\section{A. Galid}

Department of Gynaecology, Hanusch Hospital, Vienna, Austria

\section{Introduction}

From unspecific immune-stimulation in the late 19th century [1] to modern immune checkpoint modulators, immunotherapy (IOT) has evolved to become an important part of systemic anticancer treatment. Antibodies targeting proteins such as programmed cell death protein 1 (PD-1), its ligand (PD-L1), or CTLA4 counteract tumour-associated inhibition of T-cell activation and have revolutionized treatment strategies in several malignancies such as melanoma and lung cancer $[2,3]$. In contrast, the introduction of IOT in breast cancer appears markedly delayed and two factors may have contributed to this fact: Highly active and well-tolerated treatment options have been available for the treatment of hormonereceptor (HR) and HER2-positive breast cancer subtypes; in addition, breast cancer cells appear to be less immunogenic as compared with other malignant diseases $[4,5]$. On the other hand, the activity of HER2-directed monoclonal antibodies indicate that IOT in the broader sense has been highly successful in breast cancer treatment; in addition, the prognostic role of immune cells has been firmly established [6-8] suggesting that the immune response is important in breast cancer as well. The largest unmet medical need regarding novel treatment strategies exist in triple-negative breast cancer (TNBC) where currently chemotherapy is the only treatment option available. This-and the fact that TNBC seems to be the most immunogenic breast cancer subtype with a high mutational burden-led to the preferential investigation of immune checkpoint modulators in TNBC.

\section{Early trials of IOT in breast cancer}

KEYNOTE-012 was a phase Ib basket trial accruing patients with different PD-L1 positive malignancies, 
among them 32 patients with metastatic TNBC [9]. The majority of breast cancer patients were heavily pretreated and $46.9 \%$ had received more than three prior treatment lines for metastatic disease; the PD1 targeting monoclonal antibody pembrolizumab was administered at a dose of $10 \mathrm{mg} / \mathrm{kg}$ once every two weeks; here, a response rate (RR) of $18.5 \%$ (95\% CI 6.3-38.1) was observed. While progressionfree survival (PFS) was relatively short in the overall cohort, prolonged disease control was observed in patients responding to treatment (median duration of response not yet reached at the time of analysis; range 15.0 to $\geq 47.3$ weeks). Twenty-five patients with heavily pretreated PD-L1 positive oestrogen-receptor positive/HER2-negative metastatic breast cancer (MBC) were accrued to the phase Ib KEYNOTE-028 basket trial [10]. While the RR was somewhat lower as compared to TNBC (RR $12.0 \%$; 95\% CI 2.5\%-31.2\%), median duration of response was 12.0 months (range 7.4-15.9 months) suggesting that immunotherapy may offer activity in luminal disease as well. The phase Ib/II trial KEYNOTE-014 (PANACEA) included 58 pretreated HER2-positive MBC patients [11]; subjects received three-weekly trastuzumab in combination with pembrolizumab at a dose of $2 \mathrm{mg} / \mathrm{kg}$ or $10 \mathrm{mg} / \mathrm{kg}$ q3w. No dose-limiting toxicity was observed in the phase Ib part of the study; therefore, pembrolizumab was administered at the standard dose of $200 \mathrm{mg}$ q3w in the phase II extension. RR in the PD-L1 positive subset in the phase II extension part (which was defined as the primary study endpoint) was 15\% (90\% CI 7-29) and disease control rate (DCR; $\mathrm{CR}+\mathrm{PR}+\mathrm{SD} \geq 6$ months) $25 \%$ (90\% CI 14-49), respectively. Activity was markedly higher in tumours with $\geq 5 \%$ stromal TILs at baseline (RR 39\%; CDR 47\%) suggesting that in addition to PD-L1, sTILs may serve as a potential predictive marker. Of note, no clinically relevant activity was observed in the PD-L1 negative group. No cardiac events were reported and immunerelated adverse events (irAEs) were observed in $19 \%$ of all patients.

KEYNOTE-068 was a single-arm phase II trial accruing pretreated (cohort A) and treatment-naïve (cohort B) patients with metastatic TNBC; patients received single-agent pembrolizumab at a dose of $200 \mathrm{mg}$ once every three weeks. Overall, 170 patients were enrolled in cohort A; ORR was 5.3\% $(95 \%$ CI 2.7-9.9) in the entire subset and $5.7 \%$ (95\% CI 2.4-12.2) in the PD-L1-positive group [12]. Cohort B enrolled 84 patients with PD-L1 positive TNBC (defined by a combined positive score (CPS) $\geq 1$ ) without prior systemic therapy for metastatic disease; here, response rate was considerably higher (ORR of $21.4 \%$; 95\% CI 13.9-31.4) with four patients experiencing a complete response [13] suggesting that single-agent IOT may be more efficacious in early treatment lines.

Combination strategies may offer the chance to increase the activity of checkpoint inhibitors in breast cancer. As TNBC is usually regarded as chemotherapy- sensitive disease, the PD-L1 antibody atezolizumab was combined with nab-paclitaxel in a phase I study [14]; nab-paclitaxel was chosen as chemotherapy backbone as this drug requires no corticosteroid comediation. Overall, 33 patients were included; in a mixed population of pretreated and treatmentnaïve subjects, an encouraging RR of $39.4 \%$ (95\% CI $22.9 \%-57.9 \%$ ) was reported; $73 \%$ of patients experienced a grade $3 / 4$ adverse event, and seven patients (21\%) had a grade $3 / 4$ adverse events of special interest. Median overall survival (OS) was 14.7 months (95\% CI 10.1-not estimable). These favourable early results prompted further development of this combination in the phase III IMpassion130 trial. Other early phase trials evaluated the combination of IOT with different other chemotherapeutic drugs such as capecitabine or eribulin or the combination of IOT with targeted drugs such as the CDK4/6-inhibitor atezolizumab. To fully assess the benefit of IOT in these settings, results from randomized trials need to be awaited.

\section{IMpassion130}

IMpassion130 is the first prospective randomized phase III trial of immune checkpoint inhibitors in breast cancer to have results available [15]. In total, 902 patients with metastatic or locally advanced inoperable TNBC were randomized to nab-paclitaxel in combination with atezolizumab or placebo as firstline therapy. Nab-paclitaxel was administered at a dose of $100 \mathrm{mg} / \mathrm{m}^{2}$ on days 1,8 and 15 and atezolizumab at a dose of $840 \mathrm{mg}$ once every two weeks of a 28-day cycle. PD-L1 status on immune cells (ICs) was assessed by immunohistochemistry with the Ventana SP142 assay (cut-off of $\geq 1 \%$ positive ICs); $41 \%$ of all patients had centrally confirmed PD-L1-positive ICs. Co-primary endpoints were PFS and OS in the intention-to-treat (ITT) and the PD-L1+ cohorts.

In the ITT population, the combination of nab-paclitaxel with atezolizumab resulted in a PFS prolongation from 5.5 to 7.2 months (stratified HR 0.8; 95\% CI 0.69-0.92; $p=0.0025$ ); this effect was more pronounced in the PD-L1+ subset (5.0 vs. 7.5 months; HR $0.62 ; 95 \%$ CI0.49-0.78; $p<0.0001)$. In a preplanned subgroup analysis, the benefit for the combination was independent of age, prior taxane-therapy or presence of liver metastases; no effect, however, was seen in patients with PD-L1 negative tumours (HR 0.95; 95\% CI 0.79-1.15).

At the first interim OS analysis, a non-significant improvement of OS from 17.6 to 21.3 months was observed (HR 0.84; 95\% CI 0.69-1.02; $p=0.084$ ). Again, this effect was more pronounced in the PD-L1+ subset where a clinically meaningful OS prolongation of nearly 10 months from 15.5 to 25 months was reported (HR 0.62; 95\% CI 0.45-0.86). Due to hierarchical clustering of the analysis, however, this difference was formally not significant. 
Regarding key secondary endpoints, response rates and duration of response were also superior in the nab-paclitaxel plus atezolizumab arm. Overall, treatment was relatively well tolerated but a higher rate of grade 3/4 neuropathy was observed in the combination arm (6\% vs. 3\%); hypothyroidism was also more common in the IOT group $(17 \% v s .4 \%)$ but in general, irAEs were rare (pneumonitis 3\%; adrenal insufficiency $1 \%$; colitis $1 \%$ ). Any grade hepatitis was reported in $15 \%$ of patients in the atezolizumab arm and therefore not different from the placebo group (14\%).

A biomarker analysis presented at the 2018 San Antonio Breast Cancer Symposium (SABCS) showed that the vast majority of patients with PD-L1 positive tumour cells also belonged to the PD-L1 positive IC sample [16]. In addition, CD8 and TIL positive tumours had a higher rate of PD-L1 positive ICs, but neither CD8 nor TILs were predictive for IOT response in the PD-L1 negative patient subset, as was BRCA mutation status.

In summary, these data suggest a clinically meaningful additive effect of immunotherapy when added to first-line chemotherapy in TNBC patients defining a novel treatment standard in patients with PD-L1+ ICs.

\section{IOT in early stage breast cancer}

Based upon initial results of IOT in TNBC, several adjuvant and neoadjuvant studies were initiated. While no results from phase III trials have been reported so far, available data suggest that IOT may play an important role in this field as well.

I-SPY2 is platform for phase II neoadjuvant studies enabling the fast evaluation of novel agents when added to a standard chemotherapy backbone of weekly paclitaxel and doxorubicin/cyclophosphamide (AC). A positive outcome is claimed if there is an $85 \%$ Bayesian predictive probability of success of an experimental agent in a simulated 300-patient phase III trial [17]. Pembrolizumab was investigated as a component of neoadjuvant treatment in 69 patients with TNBC or high-risk luminal breast cancer [18]. In the TNBC subset, estimated pCR rates were increased from 20 to $60 \%$ with the addition of pembrolizumab; in the luminal patient cohort, corresponding numbers were 13 and $34 \%$, respectively. Of note, these figures again suggest clinical activity of checkpoint inhibitors in a non-triple negative population.

GeparNuevo is a randomized phase II trial evaluating the addition of the PD-L1 directed monoclonal antibody durvalumab to preoperative chemotherapy [19]. In total, 174 patients were randomized to receive twelve cycles of weekly nab-paclitaxel followed by four cycles of EC in combination with durvalumab or placebo. pCR was defined as the primary study endpoint which was numerically increased from 44.2 to $53.4 \%$ in the IOT group (adjusted OR 1.53; 95\% CI
$0.82-2.84 ; p=0.182$ ). Therefore, this study was formally a negative trial; a greater benefit, however, was observed in certain subsets such as patients who received durvalumab or placebo in a two-week windowof-opportunity before the initiation of chemotherapy.

Based upon the results of the Brightness study, the addition of carboplatin to standard neoadjuvant chemotherapy is often regarded as a potential standard-of-care in unselected and otherwise healthy women with early stage TNBC [20]. The phase Ib study KEYNOTE-173 therefore evaluated the addition of pembrolizumab to neoadjuvant nab-paclitaxel (cohort A) or the combination of nab-paclitaxel and carboplatin (cohort B), both followed by four cycles of AC [21]. Myelosuppression was identified as the doselimiting toxicity, while no dose-limiting toxicity linked to pembrolizumab was observed. In this study early phase study, highly encouraging pCR rates (yT0/Tis; ypN0) were reported with $60 \%(90 \%$ CI 30-85) of patients in cohort A and $90 \%$ (90\% CI 61-100) in cohort B achieving pCR.

\section{Discussion}

While the clinical development of IOT was delayed in breast cancer, available data suggest that immune checkpoint inhibitors are a valuable addition to the therapeutic armamentarium. Indeed, the IMpassion 130 trial has defined nab-paclitaxel plus atezolizumab as a potential novel first-line treatment standard in patients with metastatic TNBC and PD-L1 positive ICs; phase III trial results with other checkpoint inhibitors are expected in the near future. Other studies are focusing on combination approaches with chemotherapy, radiotherapy or biologically targeted drugs in order to increase the immunogenicity of breast cancer cells. In addition, the potential role of IOT in the (neo)adjuvant and postneoadjuant settings is currently under investigation; identification of predictive biomarkers beyond PD-L1 remains a challenge and is also required in the light of the financial burden associated with this class of drugs.

Funding Open access funding provided by Medical University of Vienna.

Conflict of interest R. Bartsch declares the following relationships: advisory role (Astra-Zeneca, Celgene, Daiichi, Eisai, Eli-Lilly, MSD, Novartis, Pfizer, Roche), lecture honoraria (Accord, Astra-Zeneca, BMS, Celgene, Eli-Lilly, Novartis, Pfizer, Roche) and research support (Novartis, Roche). E. Bergen and A. Galid declare that they have no competing interests.

Open Access This article is distributed under the terms of the Creative Commons Attribution 4.0 International License (http://creativecommons.org/licenses/by/4.0/), which permits unrestricted use, distribution, and reproduction in any medium, provided you give appropriate credit to the original author(s) and the source, provide a link to the Creative Commons license, and indicate if changes were made. 
Publisher's Note Springer Nature remains neutral with regard to jurisdictional claims in published maps and institutional affiliations.

\section{References}

1. Burdick CG. William Bradley Coley 1862-1836. Ann Surg. 1937;105(1):152-5.

2. Robert C, Long GV, Brady B, et al. Nivolumab in previously untreated melanoma without BRAF mutation. N Engl J Med. 2015;4(372):320-30.

3. Reck M, Rodríguez-Abreu D, Robinson AG, et al. Pembrolizumab versus Chemotherapy for PD-L1-Positive NonSmall-CellLungCancer. NEnglJ Med. 2016;19);375:1823-33.

4. StevenA, Seliger B. The Role of ImmuneEscape and Immune Cell Infiltration in Breast Cancer. Breast Care (Basel). 2018;1(13):16-21.

5. Bates JP, Derakhshandeh R, Jones L, Webb TJ. Mechanisms of immune evasion in breast cancer. BMC. Cancer. 2018;1(18):556

6. Denkert C, Loibl S, Noske A, et al. Tumor-associated lymphocytes as an independent predictor of response to neoadjuvant chemotherapy in breast cancer. J Clin Oncol. 2010;1(28):105-13.

7. Loi S, Sirtaine N, Piette F, et al. Prognostic and predictive value of tumor-infiltrating lymphocytes in a phase III randomized adjuvant breast cancer trial in node-positive breast cancer comparing the addition of docetaxel to doxorubicin with doxorubicin-based chemotherapy: BIG 0298. JClin Oncol. 2013;7(31):860-7.

8. Dieci MV, Criscitiello C, Goubar A, et al. Prognostic value of tumor-infiltrating lymphocytes on residual disease after primary chemotherapy for triple-negative breast cancer: a retrospective multicenter study. Ann Oncol. 2014;3(25):611-8.

9. Nanda R, Chow LQ, Dees EC, et al. Pembrolizumab in Patients With Advanced Triple-Negative Breast Cancer: Phase Ib KEYNOTE-012 Study. JClin Oncol. 2016;21);34:2460-7.

10. Rugo HS, Delord JP, Im SA, et al. Safety and Antitumor Activity of Pembrolizumab in Patients with Estrogen Receptor-Positive/Human Epidermal Growth Factor Receptor 2-Negative Advanced Breast Cancer. Clin Cancer Res. 2018;12);24:2804-11.

11. Giobbie-Hurder LSA. Gombos A. December: et al. Phase $\mathrm{Ib} / \mathrm{II}$ study evaluating safetyand efficacy of pembrolizumab and trastuzumab in patients with trastuzumab-resistant HER2-positive advanced breast cancer: results from the PANACEA study (IBCSG 45-13/BIG 4-13/KEYNOTE-014). Abstr. GS2-06; presented at the 2017 San Antonio Breast Cancer Symposium; 2017. San Antonio, Texas, USA.

12. Adams S, Schmid P, Rugo HS, et al. Pembrolizumab Monotherapy for Previously Treated Metastatic Triple-Negative Breast Cancer: Cohort A of the Phase 2 KEYNOTE-086
Study. Ann Oncol. 2018. https://doi.org/10.1093/annonc/ mdy517

13. Adams S, Loi S, Toppmeyer D, et al. Title: Pembrolizumab Monotherapy for Previously Untreated, PD-L1-Positive, Metastatic Triple-Negative Breast Cancer: Cohort B of the Phase 2 KEYNOTE-086 Study. Ann Oncol. 2018. https:// doi. org/10.1093/annonc/mdy518

14. Adams S, Diamond JR, Hamilton E, et al. Atezolizumab Plus nab-Paclitaxel in the Treatment of Metastatic TripleNegative Breast Cancer With 2-Year Survival Follow-up: A Phase 1b Clinical Trial. JAMA Oncol. 2018. https://doi.org/ 10.1001/jamaoncol.2018.5152

15. Schmid P, Adams S, Rugo HS, et al. Atezolizumab and Nab-Paclitaxel in Advanced Triple-Negative Breast Cancer. NEngl J Med. 2018;379(22):2108-21.

16. Emens LA, Loi S, Rugo HS, et al. IMpassion130: Efficacy in immune biomarker subgroups from the global, randomized, double-blind, placebo-controlled, phase III study of atezolizumab + nab-paclitaxel in patients with treatmentnaïve, locally advanced or metastatic triple-negative breast cancer. Abstr. GS1-04; presented at the 2018 San Antonio Breast Cancer Symposium, December 2018, San Antonio, Texas, USA.

17. Rugo H, Olopade OI, DeMichele A, et al. Adaptive Randomization of Veliparib-Carboplatin Treatment in Breast Cancer. NEngl J Med. 2016;375(1):23-34.

18. Nanda R, Liu MC, Yau C, et al. Pembrolizumab plus standard neoadjuvant therapy for high-risk breast cancer (BC): Results from I-SPY2. Abstr. 506; presented at the 2017 ASCO Annual Meeting, June 2017, Chicago, Illinois, USA.

19. Loibl S, Untch M, Burchardi N, et al. Randomized phase II neoadjuvant study (GeparNuevo) to investigate the addition of durvalumab to a taxane-anthracycline containing chemotherapy in triple negative breast cancer (TNBC). Abstr. 104; presented at the 2018 ASCO Annual Meeting, June 2018, Chicago, Illinois, USA.

20. Loibl S, O'Shaughnessy J, Untch M, et al. Addition of the PARP inhibitor veliparib plus carboplatin or carboplatin alone to standard neoadjuvant chemotherapy in triplenegative breast cancer (BrighTNess): a randomised, phase 3 trial. Lancet Oncol. 2018;19(4):497-509.

21. Schmid P, ParkYH, Muñoz-Couselo E, et al. Pembrolizumab (pembro) + chemotherapy (chemo) as neoadjuvant treatment for triple negative breast cancer (TNBC): Preliminary results from KEYNOTE-173. Abst. 556; presented at the 2017 ASCO Annual Meeting, June2017, Chicago, Illinois, USA.

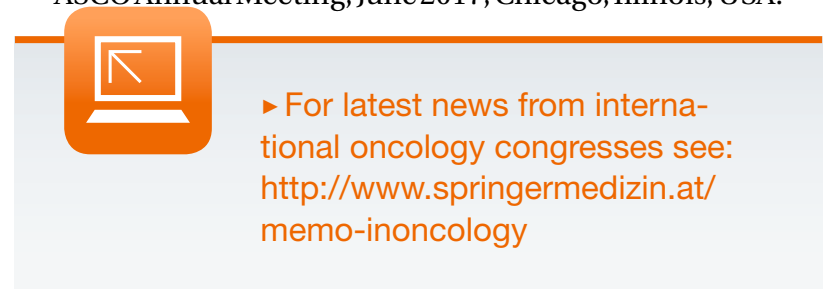

